\title{
2D Numerical Simulation and Sensitive Analysis of H-Darrieus Wind Turbine
}

\author{
Seyed Mohammad E. Saryazdia and Mehrdad Boroushaki ${ }^{{ }^{*}}$ \\ a PhD candidate in Energy Systems Engineering, Sharif University of Technology, Tehran, P.O.Box 14565-114, Iran \\ ${ }^{b}$ Associate Professor of Department of Energy Engineering, Sharif University of Technology, Tehran, P.O.Box 14565-114, Iran
}

\begin{abstract}
Recently, a lot of attention has been devoted to the use of Darrieus wind turbines in urban areas. The aerodynamics of a Darrieus turbine are very complex due to dynamic stall and changing forces on the turbine triggered by changing horizontal angles. In this study, the aerodynamics of H-rotor vertical axis wind turbine (VAWT) has been studied using computational fluid dynamics via two different turbulence models. Shear stress transport (SST) k- $\omega$ turbulence model was used to simulate a 2D unsteady model of the H-Darrieus turbine. In order to complete this simulation, sensitivity analysis of the effective turbine parameters such as solidity factor, airfoil shape, wind velocity and shaft diameter were done. To simulate the flow through the turbine, a $2 \mathrm{D}$ simplified computational domain has been generated. Then fine mesh for each case consisting of different turbulence models and dimensions has been generated. Each mesh in this simulation dependent on effective parameters consisted of domain size, mesh quality, time step and total revolution. The sliding mesh method was applied to evaluate the unsteady interaction between the stationary and rotating components. Previous works just simulated turbine, while in our study sensitivity analysis of effective parameters was done. The simulation results closely match the experimental data, providing an efficient and reliable foundation to study wind turbine aerodynamics. This also demonstrates computing the best value of the effective parameter. The sensitivity analysis revealed best value of the effective parameter that could be used in the process of designing turbine. This work provides the first step in developing an accurate 3D aerodynamic modeling of Darrieus wind turbines.
\end{abstract}

Keywords: Darrieus, VAWT, CFD, Dynamic stall, Sensitive analysis

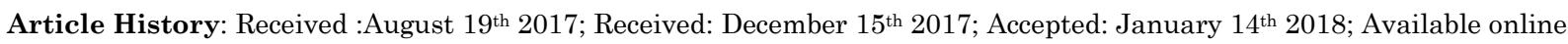

How to Cite This Article: Saryazdi, S. M. E. and Boroushaki, M. (2018) 2D Numerical Simulation and Sensitive Analysis of HDarrieus Wind Turbine. Int. Journal of Renewable Energy Development,7(1),23-34

https://doi.org/10.14710/ijred.7.1.23-24

\section{Introduction}

The energy crisis that developed over the last century has arisen plenty of economic problems and has instigated large-scale research in the field of wind power. Recent advances in technology and research have proved wind energy is an environmental friendly and economical energy resource. Two main types of wind turbines exist based on rotation axis and operation: a vertical axis wind turbine (VAWT) and a horizontal axis wind turbine (HAWT). The prevalent type is the horizontal axis wind turbine often seen scattered across the landscape in areas of relatively level terrain with predictable year-round wind conditions. The main subject of research in wind turbines has focused on HAWT for decades, largely because they share common operation and dynamics with rotary aircraft. The result has been large and advanced HAWTs. The other main type of wind turbine is the vertical axis wind turbine (VAWT). This type of wind turbine rotates about an axis that is perpendicular to the oncoming wind/air flow. Therefore, it can use wind coming from any direction for producing energy.

VAWTs consist of two major types, the Darrieus rotor, and the Savonius rotor. The Darrieus wind turbine is a VAWT that rotates around a central axis due to the lift produced by the rotating airfoils, whereas a Savonius rotor rotates due to the drag created by its blades. Two different types of Darrieus exist, the H-rotor and the eggbeater type rotor. The $\mathrm{H}$ rotor Darrieus has high power efficiency and works well in turbulent wind, thus it is suitable for urban areas. Figure 1 shows the four different types of wind turbines, this work focuses on the H-rotor Darrieus wind turbine.

In a review of the literature, it was found that four models were applied to study the aerodynamic performance of H-rotors: Viscous model (CFD),

\footnotetext{
*Corresponding author: boroushaki@sharif.edu
} 
Momentum model, Local circulation model, and Vortex model. Each model has its advantages and disadvantages.
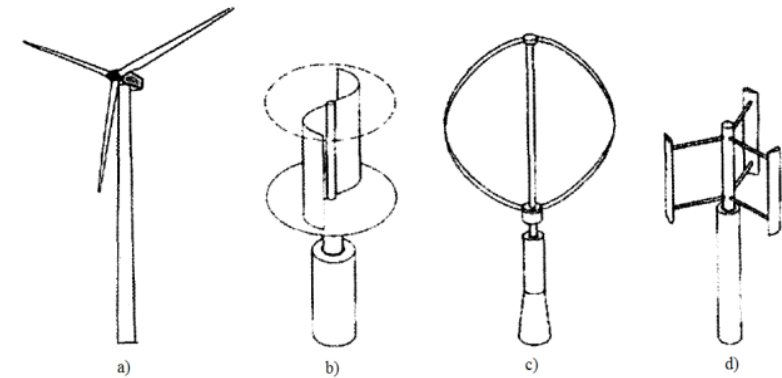

Fig. 1 Main types of wind turbines a) HAWT b) Savonius c) egg beater Darrieus d) H-rotor Darrieus

The main advantage of momentum model is the smaller amount of necessary computer time compared to the other approaches (Spera, 2009). Before advances were made in computer technology, complex problems, such as the airflow around the H-rotors, was studied using empirical aerodynamic models developed based on data from wind tunnel experiments. These models have continued to improve as more data have become available. While empirical models are still used today, the development of computers and algorithms has led to the concurrent application of computational fluid dynamics (CFD) in the research of H-rotors. Because of the complexity of the unsteady aerodynamics of the H-rotor, CFD methods have become an attractive way to analyze aerodynamic performance. The CFD method is considered the baseline for other models because it is complete and general. The fluid field captured around the wind turbine provides excellent data for analysis. In addition, instantaneous flow characteristics can be obtained from the unsteady-state model. In this study, we used CFD techniques to study the aerodynamic performance of an H-rotor with SST k- $\omega$ turbulent model.

Allet and Paraschivoiu (1995) discretized a steady, incompressible Navier-Stokes equation in cylindrical coordinates with the control volume approach. The CFD results were compared with the DMSM results and experimental data. The dynamic stall effects were considered in both models as well as both CFD simulations, and DMSM simulation were able to predict the power coefficient accurately at low tip speed ratio (TSR), where the dynamic stall occurred. However, when it came to instantaneous data, CFD simulation offered a much better prediction. For higher TSRs, the CFD simulation also performed better than the DMSM simulation. Horiuchi et al. (2005) used detached eddy simulation (DES) to numerical simulation of wind turbine. They found that the wind velocity could not recover in $\mathrm{x} / \mathrm{r}=10$ downstream, so this constraint must be considered when installing wind turbine in large quantity. Guerri (2007) examined the aerodynamic performance of a small rotating vertical axis wind turbine by using a
RANS solver and moving mesh technique. The SST k$\omega$ model was applied to the $2 \mathrm{D}$ simulation. Comparison between CFD and multiple stream tube models was carried out, based on blade forces and torques obtained from integrating the pressure and shear stress over the blade surface was done. Besides, it was found that the rotational speeds predicted by CFD model were less than those of the multiple stream-tube theory were. The results showed that the CFD technique gave a good evaluation of wind turbine performances and remained sensitive to the airfoil section data. Hamada (2008) studied H-rotor turbine with RNG-k- $\varepsilon$ turbulence model. The $2 \mathrm{D}$ unsteady simulation showed that negative torque was generated at azimuth angle equal to $0^{\circ}$ and the minimum value of torque reached at azimuth angle $13^{\circ}$. After that, torque increased as lift direction became more favorable for positive torque contribution. As a result of increasing the angle of attack, more lift was generated. At azimuth angle equal to $90^{\circ}$, positive torque reached the maximum value. After then, the torque decreased as the drag increased until dynamic stall happened. At azimuth angle equal to $180^{\circ}$, the torque reached a negative value again because of drag becoming dominant. In the downwind direction, a smaller positive torque was generated. As the variation in both the direction and the magnitude of the relative velocity vector, the dynamic stall behavior of the VAWT blade was different from that of a pitching airfoil. Hwang et al. (2009) investigated 2D H-rotor with both CFD and

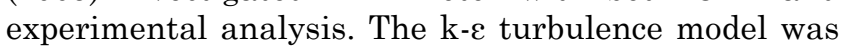
used in CFD simulation. The power coefficient reached its maximum at a lower tip speed ratio compared with experiment. By optimization of the individual blade control, the performance was improved by $25 \%$.

EI-Samanoudy (2010) reported the effect of some design parameters on the performance of H-rotors by both wind tunnel experiment and CFD modeling. The pitch angle of their wind turbine varied from $-10^{\circ}$ to $60^{\circ}$. The performance of the wind turbine increased as the pitch angle increased. After arriving at peak performance, the power coefficient started to decrease. The effect of blade number was also investigated. The wind turbine performance was increasing significantly when the blade number increased from two to three; however, only a slight increase was obtaining when the blade number became four. Howell (2010) studied the performance of a small scale VAWT with both wind tunnel and CFD modeling. The experiments showed that the surface roughness on the turbine rotor blades had a great effect on the rotor performance. The investigation of two bladed and three bladed H-rotors showed that the turbine power coefficient was significantly higher for the rotors with higher solidity. The k- $\varepsilon$ turbulence model was used in 2D simulation. Shengyi Wang et al. (2010) studied the dynamic stall phenomenon in an oscillating blade by performing a numerical investigation on a VAWT turbine with NACA 0012 blade at a low Reynolds 
number (Re=105). Results showed good agreement with the numerical study as well as standard and SST $\mathrm{k}-\omega$ turbulence models were considered. They showed that the SST model was better at the lower angles of attack. At the same time at angles of attack greater than $\left(24^{\circ}\right)$ in remote areas, neither model provided good results for current flow. While the standard $\mathrm{k}-\omega$ model predicts the current flow better on the blade, it did not predict the correct velocity profile. In general, the SST k- $\omega$ was found to provide a more complex model relative to the standard $\mathrm{k}-\omega$ model.

It should be noted that previous research focused primarily on CFD simulation of H-rotor turbines with various specifications, performance parameters, and turbulence model. However, effects of different design parameter on the performance of turbine were not considered. In this research, important performance parameters of turbine were studied in order to identify the impact of each factor on the performance of the turbine. This study can be used to design turbine in different situations. Solidity factor, airfoil shape, wind velocity, and shaft diameter parameters have been chosen to study their effects on turbine performance in this study. In order to obtain the best power performance of the H-rotor, power coefficients were calculated at various TSRs. A physical flow field may be represented in terms of a mathematical model. The models must observe the fundamental properties of a flow field, including conservation of mass, momentum and energy. Computational fluid dynamics use various discretization schemes to approximate the governing equations of the flow field. The flow field is divided into a certain number of cells, which are called grids. Then the equations are solved at discrete grid points at each time step. By solving the governing equations simultaneously, the solution of the flow field can be obtained, while the CFD simulation has been implemented by the CFD code. To achieve optimal mesh and mesh-independent solutions, each model's mesh passed some independent tests, and the results are given below.

\section{Methodology}

\subsection{Aerodynamic features of $H$-rotor blades}

The cross section of an H-rotor Darrieus wind turbine is shown in Figure 2, including the directions of the lift and drag, and their normal, tangential, y, and $\mathrm{x}-$ direction force components. On a rotating H-rotor, the forces can be expressed in tangential and normal directions. The tangential force can be obtained from the difference between the tangential component of lift and drag forces, while the normal force perpendicular to the rotating direction is equal to the difference between their normal components. The tangential force $F_{t}$ and the normal force $F_{n}$ can be expressed as below:

$$
\begin{aligned}
& F_{t}=L \sin \alpha-D \cos \alpha \\
& F_{n}=L \cos \alpha-D \sin \alpha
\end{aligned}
$$

where $\mathrm{L}$ is the lift power and $\mathrm{D}$ is the drag power. Total forces of the turbine can be obtained in $\mathrm{x}$ and $\mathrm{y}$ directions as expressed below:

$$
\begin{aligned}
& F_{x}=F_{t} \cos \theta-F_{n} \sin \theta \\
& F_{y}=F_{n} \cos \theta+F_{t} \sin \theta
\end{aligned}
$$

The force coefficients can be expressed as:

$$
\begin{aligned}
& C_{L}=\frac{L}{1 / 2 \rho c V_{\infty}^{2}} \\
& C_{D}=\frac{D}{1 / 2 \rho c V_{\infty}^{2}} \\
& C_{t}=\frac{F_{t}}{1 / 2 \rho c V_{\infty}^{2}} \\
& C_{n}=\frac{F_{n}}{1 / 2 \rho c V_{\infty}^{2}} \\
& C F S=\frac{F_{y}}{1 / 2 \rho c V_{\infty}^{2}}
\end{aligned}
$$

Where $C_{L}$ is the lift coefficient, $C_{D}$ is the drag coefficient, $C_{n}$ is the normal force coefficient, $C_{t}$ is the tangential force coefficient, and CFS is the y-direction force coefficient, respectively. The AOA ( $\alpha$ ) is the angle between relative wind velocity and the blade rotating direction (Figure 2).

In Figure 2, the $\mathrm{W}$ vector shows the relative velocity and is equal to the vector sum of free stream velocity and rotating velocity, and $\alpha$ is the angle of attack. Since the angle of attack and relative wind speed changes with the azimuth positions, the variable forces can be considered as a function of the azimuth angle. The relative wind speed can be obtained by the vector sum of inlet wind velocity and rotating velocity. At different TSR ( $\lambda$ ), due to changes in the AOA (a) and the relative wind speed, the blade exhibits various aerodynamic performance in each cycle. The AOA (a) will not show much change when the TSR increases. The relationship between the AOA (a) and TSR is expressed in Equation (10). 


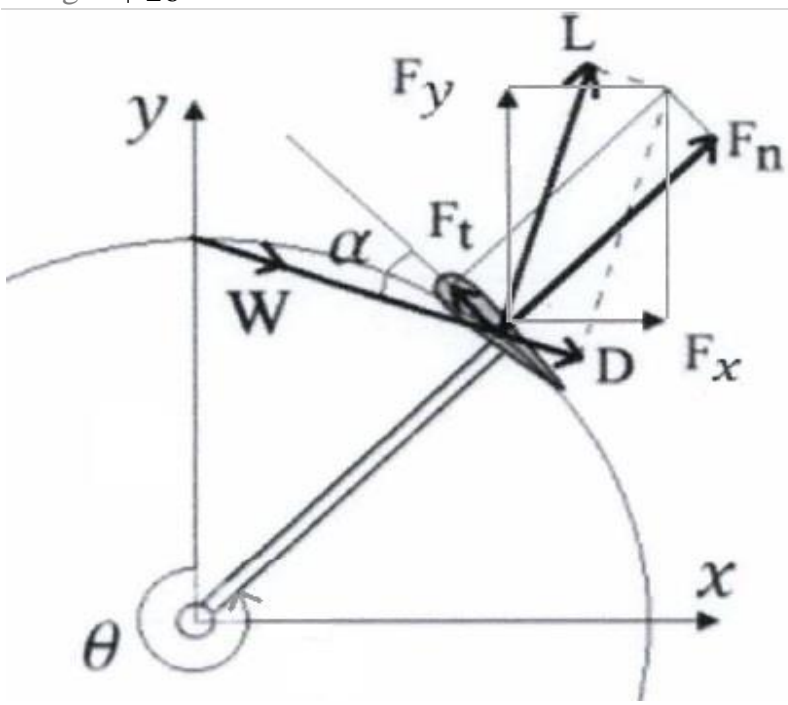

Fig. 2 Force diagram of an H-rotor Darrieus turbine

$\alpha=\tan ^{-1} \frac{\sin \theta}{\cos \theta+\lambda}$

Where $\theta$ is the azimuth angle and $\lambda$ is the TSR.

The five necessary non-dimensional parameters to be considered in designing H-rotor are as follows:

a) The ratio of the blade liner tip speed and the wind speed is defined as (TSR):

$$
\lambda=T S R=\frac{n R}{V_{\infty}}
$$

b) The solidity factor of the turbine is defined as:

$$
\sigma=\frac{N c}{2 R}
$$

c) The aspect ratio is defined as:

$$
a=\frac{H}{R}
$$

d) The Reynolds number base on the chord length is defined as:

$$
\operatorname{Re}=\frac{\rho V_{\infty} c}{\mu}
$$

e) The power coefficient $(\mathrm{Cp})$ is defined as:

$$
C_{P}=\frac{P}{1 / 2 \rho A V_{\infty}^{3}}
$$

Where A represents the cross-sectional area of the wind turbine. This coefficient has the limitation of
0.593, which is known as Betz constant (Betz, 1920). Text should be produced within the dimensions shown on these pages. Make use of the maximum stipulated length apart from the following two exceptions: (i) do not begin a new section directly at the bottom of a page, but transfer the heading to the top of the next column; (ii) you may exceed the length of the text area by one line only in order to complete a section of text or a paragraph. You must use 1.0 (single) line spacing and Cambria font. However, when typing complicated mathematical text it is important to increase the space between the text lines in order to prevent suband superscript fonts overlapping one another and making your printed matter illegible. If you are using a desktop publishing program ensure that your auto adjust for interline spacing is suitably set to prevent overlapping but without leaving too much space.

\subsection{Governing Equation}

In the fluid flow analysis, the compressibility of the flow will be considered when the Mach number is larger than 0.3. The Mach number of flow around the $\mathrm{H}$-rotor is much smaller than 0.3 , so the flow will be considered as incompressible in the analysis. Therefore, the density of the flow will be constant.

The turbulence convected and dissipated in the flow field is generated due to various factors. The transported quantities including momentum and energy in momentum transfer as well as various concentrations in mass transfer are mixed in the turbulence due to the fluctuating velocity field. The fluctuations contain various scales and frequencies. Finer grids are required to capture the small-scale fluctuations with high frequency.

Reynolds Averaged Navier-Stokes (RANS) equations are used to solve the incompressible flow using a Boussinesq approach, which relates the Reynolds stress incurred by averaging the flow equations to the mean velocity gradients. In Reynolds averaging, the solution variables in instantaneous Navier-Stokes equations are divided into the mean and fluctuating components. For the velocity components:

$u_{i}=\bar{u}_{l}+u_{l}^{\prime}$

where $\bar{u}$ is the mean velocity components and $u^{\prime}$ is the fluctuating velocity components ( $\left.i=1,2,3\right)$. Likewise, for pressure and other scalar quantities:

$\phi=\bar{\phi}+\phi_{l}^{\prime}$

where $\phi$ represents a scalar such as pressure, energy, or species concentration. Substituting expressions of this form, the Reynolds-averaged Navier-Stokes equations (RANS) can be obtained. 
$\frac{\partial \bar{u}_{l}}{\partial x_{i}}=0$

$\rho\left(\frac{\partial \bar{u}_{l}}{\partial t}+\bar{u}_{J} \frac{\partial \bar{u}_{l}}{\partial x_{i}}\right)=-\frac{\partial \bar{p}}{\partial x_{i}}+\mu\left(\frac{\partial^{2} \bar{u}_{l}}{\partial x_{j} \partial x_{j}}\right)-\frac{\partial}{\partial x_{j}}\left(\rho \overline{u_{l}^{\prime} u_{J}^{\prime}}\right)$

These equations have the same general form as the instantaneous Navier-Stokes equations, with $u_{J}$ representing the $\mathrm{j}$ component of mean velocity, $\mu$ the viscosity, and $\mathrm{x}_{\mathrm{i}}$ the $\mathrm{j}$ coordinate. The Reynolds stresses $\rho \overline{u_{l}^{\prime} u_{J}^{\prime}}$, must be modeled to close Equation (19). The SST k- $\omega$ model is another turbulence model used in this study. This model is similar in form to the standard k- $\omega$ model. The transport equations derived from turbulence kinetic energy (k) and turbulence frequency rate $(\omega)$ in SST k- $\omega$ are shown below:

$$
\begin{aligned}
& \frac{\partial(\rho k)}{\partial t}+\frac{\partial}{\partial x_{i}}\left(\rho k u_{i}\right)=\frac{\partial}{\partial x_{j}}\left(\Gamma_{k} \frac{\partial k}{\partial x_{i}}\right)+\tilde{G}_{k}-Y_{k}+S_{k} \\
& \frac{\partial}{\partial t}(\rho \omega)+\frac{\partial}{\partial x_{i}}\left(\rho \omega u_{i}\right)=\frac{\partial}{\partial x_{j}}\left(\Gamma_{\omega} \frac{\partial \omega}{\partial x_{i}}\right)+G_{\omega}-Y_{\omega}+D_{\omega}+S_{\omega}
\end{aligned}
$$

where $\mathrm{G}_{\mathrm{k}}$ represents the generation of turbulent kinetic energy due to the mean velocity gradients, calculated from $G_{k} . G_{\omega}$ is the generation of $\omega$, calculated as described for the standard k- $\omega$ model. $\Gamma_{\mathrm{k}}$ and $\Gamma_{\omega}$ represent the effective diffusivity of $\mathrm{k}$ and $\omega$. $Y_{k}$ and $Y_{\omega}$ represent the dissipation of $k$ and $\omega . S_{k}$ and $\mathrm{S}_{\omega}$ are user-define source terms (Launder and Spalding, 1972).

\subsection{Geometry and mesh generation}

So as to reduce the computation time, a 2-D calculation without rotor shaft was carried out. Based upon the literature review (Wang et al. 2010), Darrieus turbine with certain features listed in the Table 1, was considered for simulation.

To simulate the flow through the turbine, a 2-D simplified computational domain was generated, as shown in Figure 3. Different domains with distinct mesh were tested to achieve a fine mesh. The domain consists of three subdomains each divided by interface boundaries.

A rotating domain or ring is located between two stationary domains; the large stationary rectangular domain is $40 \mathrm{~m}$ in crosswind width by $50 \mathrm{~m}$ in stream wise length with the interior circle (see Figure 3). The inlet and side surfaces of the large stationary domain were fixed both at velocity inlet. In order to keep the solution results independent from the domain size, the boundary conditions were located far from the turbine blades. A wall type boundary condition was defined for the blades. The surface of the blades was modeled as a smooth with no-slip surfaces. The right line of the large stationary sub-domain was defined as the pressure outlet.

Table1.

Experimental Rotors Features

\begin{tabular}{cc}
\hline \hline Design parameter & Value \\
\hline Rotor radius $(\mathrm{m})$ & 0.5 \\
Blade length $(\mathrm{m})$ & 1.03 \\
Blade chord(mm) & 85 \\
TSR & $1,2,2.5,3$ \\
Number of blades & 3 \\
Blade type & NACA0021 \\
\hline \multicolumn{1}{c}{ Air constants } & \\
\hline Air density $\left(\mathrm{kg} / \mathrm{m}^{\wedge} 3\right)$ & 1.225 \\
Wind speed $(\mathrm{m} / \mathrm{s})$ & 6 \\
Turbulence intensity & $5 \%$ \\
& \\
\hline
\end{tabular}

Source: RacitiCastelli et al. (2011)

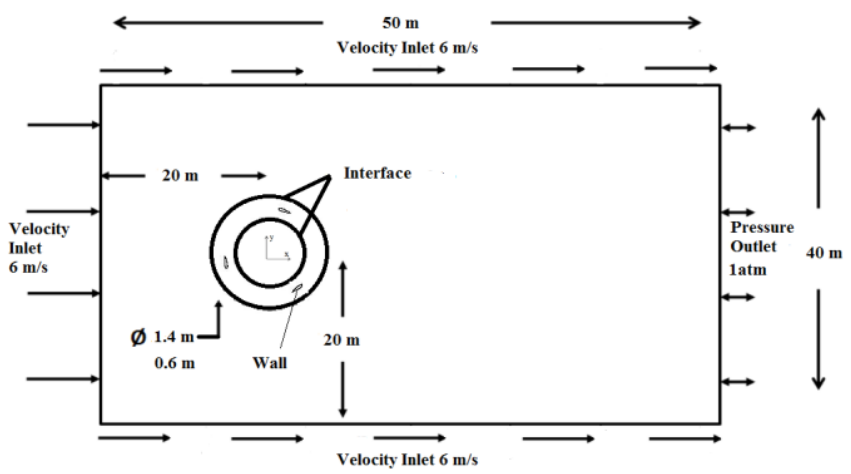

Fig. 3 Computational domain and boundary conditions to simulate the current around an H-rotor Darrieus wind turbine

The mesh generation was carried out using a suitable mesh generation code. Figure 4 depicts the generated fine mesh for the problem domain. The moving mesh formulation would be applied to the rotating area to approximate the motion of airfoil blades. An unstructured mesh was used in the free-stream region of the domain, resulting in close to745,000 nodes for the SST k- $\omega$ model.

A highly refined mesh was used in the close vicinity of the blades, resulting in a total of 300 elements over the surface of each blade and 40 levels of cells in the boundary layer for the SST k- $\omega$ model. The fine mesh structure at the boundary layer for both turbulent models was depicted in Figure 5.

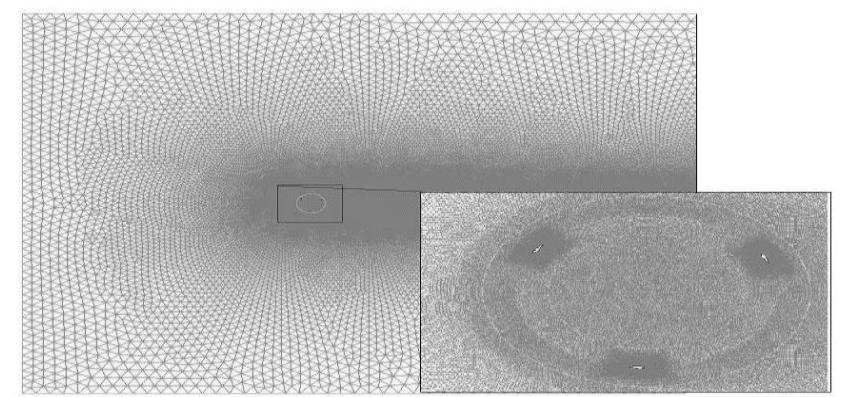

Fig. 4 Generated mesh structure in the domain of the H-rotor Darrieus turbine 


\subsection{Mesh validation}

In modeling of turbine calculation, when the simulation has converged, the $\mathrm{y}^{+}$parameter is calculated as follows to show the quality results and also the mesh generated (RacitiCastelli et al. 2011).

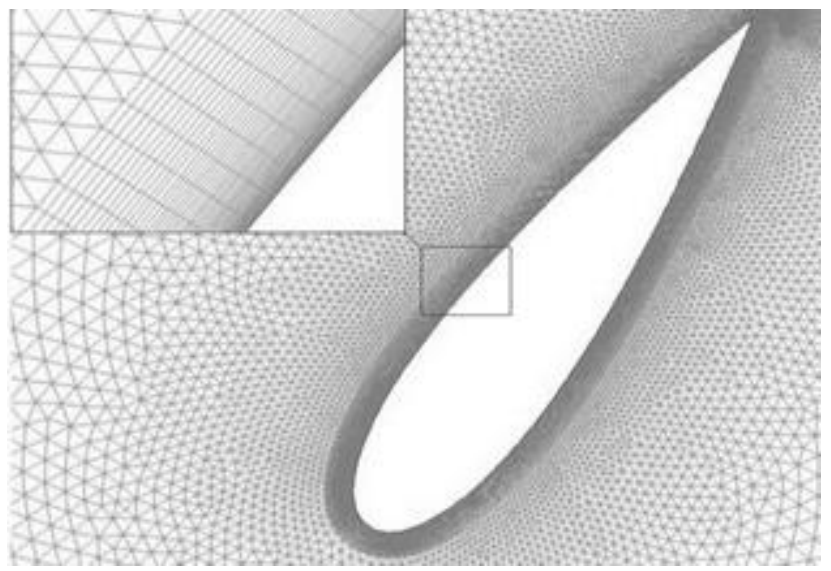

Fig. 5 Generated mesh structure near blades for the SST k- $\omega$ turbulent models

$y^{+}=y \frac{\sqrt{\frac{\tau \omega}{\rho}}}{v}$

Where $\mathrm{y}$ is the distance to the nearest wall. For a reasonable calculation, the $\mathrm{y}+$ should be less than the one for the SST k- $\omega$ (Menter et al. 1990), and between 30 to 100 for the k- $\varepsilon$ (White et al.) models. Depending on the post processing, the value of $\mathrm{y}+$ for the blades was in very good agreement with the aboverecommended values and is shown in Figure 6 (Menter et al. 1990).

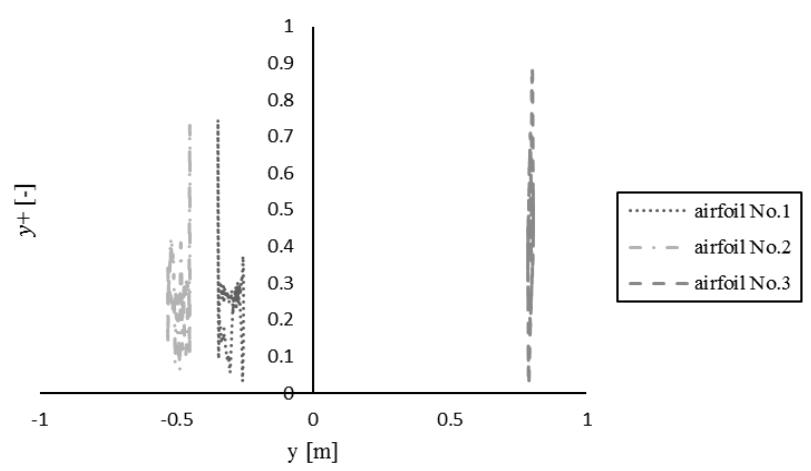

Fig. 6 The value of $y+$ for three blades, calculated with the SST $\mathrm{k}-\omega$ turbulent model in an H-rotor Darrieus turbine.

The criteria considered in this study for presenting the quality of the results was the cross stream force coefficient (CSFC), which is aligned with the $y$ direction in Figure 2. Figure 7 shows that the first two rotations are considerably affected by the initial conditions. The power coefficient and maximum CSFC predicted by the 3rd, 4th and 5th revolutions differ by less than 0.1 and $0.5 \%$, respectively, and revealed the solution converged to a stable condition. Thus, the third revolution data was used for rest of the study.

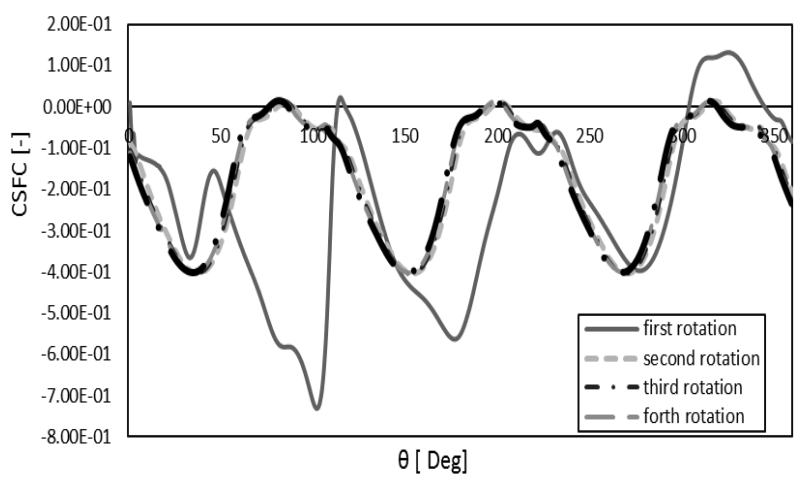

Fig. 7 CSFC of the total turbine per rotation

\subsection{Solver Setting in CFD code}

Numerical simulation of the flow field was carried out using hybrid finite volume/finite-element methods of CFD code. The code has to solve the incompressible unsteady Reynolds-averaged Navier-Stokes equations throughout the entire flow domain. The solver was fixed as pressure based. A pressure-based solver employs an algorithm which belongs to a general class of methods called the projection method. In the projection method, the constraint of mass conservation (continuity) of the velocity field is achieved by solving a pressure (or pressure correction) equation. The pressure equation is derived from the continuity and the momentum equations in such a way that the velocity field, corrected by the pressure, satisfies the continuity. Since the governing equations are nonlinear and coupled to one another, the solution process involves iterations wherein the entire set of governing equations is solved repeatedly until the solution converges. The sliding mesh model was activated by setting the angular velocity in a ring filed and attach airfoils to this field. The motion of the moving mesh was specified with a certain angular velocity determined by the TSR.

Pressure-velocity coupling is achieved to derive an additional condition for pressure by reformatting the continuity equation. The pressure-based solver allows you to solve the flow problem in either a segregated or a coupled manner. The Pressure-Implicit with Splitting of Operators (PISO) velocity-pressure coupling scheme was used to solve the hydrodynamic equation. A second order upwind spatial discretization algorithm was used for all equations (pressure, momentum, and turbulence). A second order implicit formulation was employed for Transient terms in addition to the least squares cell based algorithm set for gradients. Convergence checking was performed with two control monitors, a monitor for residuals (continuity, momentum, and turbulence) and a monitor for a lift coefficient on blades. Convergence 
criteria were fixed to $10^{-5}$ for all equations. In the process of choosing an appropriate time step size, $12^{*} 10^{-3}$ second was found to be the best time step size. In this time step size, the act of turbine in each degree can be achieved. Note the resulting Reynolds number based on equation (14) is 32,000 .

\section{Simulation Results}

Figures 8 show contours of velocity in SST k- $\omega$ turbulence models, when the TSR was equal to 2 . Thus, in the post processing, we focused more on results from SST k- $\omega$ at a TSR equal to 2 . As can be seen, this $2 \mathrm{D}$ simulation is able to predict stall and wake effects. Depending on angular position and motion of the blades, each blade produced a different type of turbulence.

Figure 9 shows the velocity vector around one blade during one full rotation. It can be seen that the blade underwent a leading edge dynamic stall (L-1) at angle $\theta=285^{\circ}$ followed shortly by a trailing edge dynamic stall $(t-1)$ at the suction side of the airfoil (Figure 9-a\&b). The leading edge vortex up to angle $\theta=75^{\circ}$ developed and then detached from the surface and shed into the wake (Figure 9 a- b \& c). The trailing edge vortex attaches to the surface because of wind velocity up to angle $\theta=90^{\circ}$ and then disappeared (Figure 9-c).

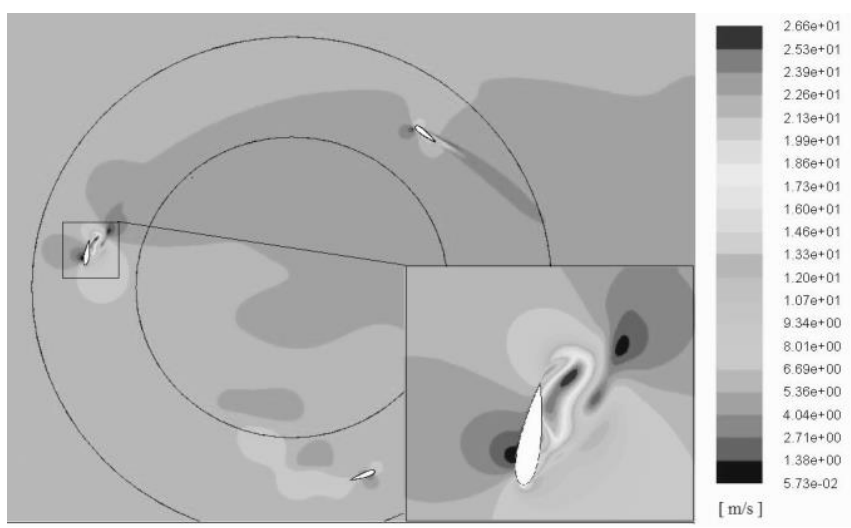

Fig. 8 Contours of velocity magnitude in SST k- $\omega$ at $\lambda=2$, at $\theta=85^{\circ}$

The flow will reattach from the surface of the blade up to angle $\theta=285^{\circ}$. While at an angle of $\theta=220^{\circ}$, the leading vortex is shed to wake incidence with airfoil (Figure 9- d). This cycle is repeated in each blade rotation and then detached from the surface and shed into the wake (Figure $9 \mathrm{a}-\mathrm{b} \& \mathrm{c}$ ). The trailing edge vortex attaches to the surface because of wind velocity up to angle $\theta=90^{\circ}$ and then disappeared (Figure 9- c). The flow will reattach from the surface of the blade up to angle $\theta=285^{\circ}$. While at an angle of $\theta=220^{\circ}$, the leading vortex is shed to wake incidence with airfoil (Figure 9- d). This cycle is repeated in each blade rotation.
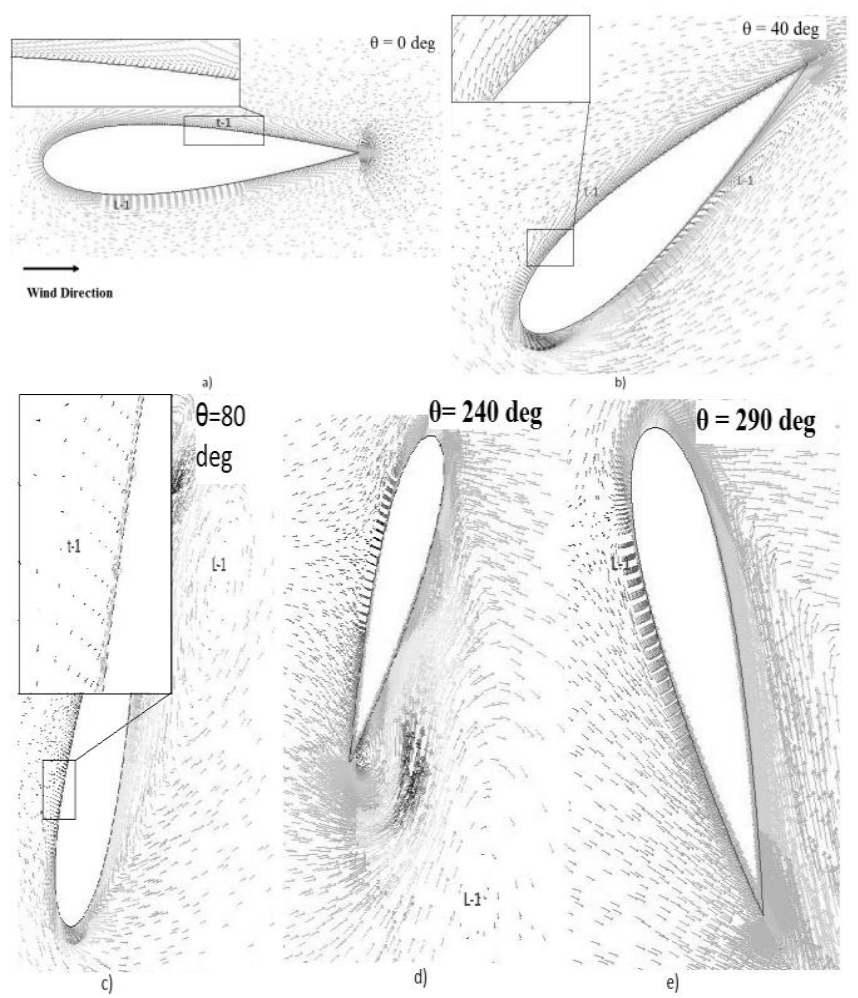

Fig. 9 Velocity vector in a single rotation of one blade at TSR=2 using SST k- $\omega$ turbulence model a) current flow around the airfoil at position $\theta=0^{\circ}$ with two vortexes (t-1) and (L-1); b) current flow around the airfoil at position $\theta=40^{\circ}$ with two vortex (t-1) and (L-1); c) current flow around the airfoil at position $\theta=80^{\circ}$ with two vortex $(\mathrm{t}-1)$ and (L-1) where (t-1) starts to disappear and (L-1) separates from surface; d) current flow around the airfoil at position $\theta=240^{\circ}$, incidence between (L-1) and airfoil; and e) current flow around the airfoil at position $\theta=290^{\circ}$ where vortex ( L-1) starts to exist.

Figure 10 depicts the velocity magnitude at the middle of the turbine and at 3 distances behind the turbine location with a $\mathrm{TSR}=2$. The results showed significant flow momentum extraction occurring through the turbine; reducing the flow velocity by $40 \%$ at the center line of the turbine (Mid Turbine), 30\% at $10 \mathrm{~m}$, and $8 \%$ at $20 \mathrm{~m}$. The effect of the turbine beyond $30 \mathrm{~m}$ was negligible. Figure 10 shows an incredible increase in air velocity at the centerline, caused by the velocity of the blade at the centerline at that moment.

Figure 11 presents the CL versus a curve at TSR equal to 2. The left side shows the results attained from this simulation, and for comparison, the right side shows the CFD results of Wang et al. 2010. The curves in Figure 11 have some differences, these differences arising from the fact that Wang et al. 2010 , simulated only one oscillating airfoil, while in this study a turbine with three blades was simulated. On the other hand, the final results are more realistic and fluctuated less than the CFD simulation conducted by Wang et al. (2010).

This curve shows good agreement with experimental data (Lee and Gerontakos 2004), dashed line on Figure 11b. Dynamic stall is an unsteady 
Citation: Saryazdi, S. M. E. and Boroushaki, M. (2018) 2D Numerical Simulation and Sensitive Analysis of H-Darrieus Wind Turbine. Int. Journal of Renewable Energy Development, 7(1),23-34, doi.org/10.14710/ijred.7.1.23-34

$\mathrm{P}$ a g e $\mid 30$

aerodynamic effect that occurs at low tip speed ratios, due to rapid and large variations of the angle of attack a vortex originates on the leading edge of the airfoil; this vortex produces higher lift, drag, and pitching moment coefficients than those produced in a static stall. The effect of dynamic stall can be seen in Figure 11a. Dynamic stall keeps the value of CL from a decreasing trend at $A O A=5^{\circ}$. The second peak, in the lift coefficient versus AoA curve, indicates the appearance of an incidence between the leading edge shed from the upstream pass and airfoil (seen in Figure 9-d). This peak occurred at $A O A=27^{\circ}$ and hindered the decrease lift coefficient.

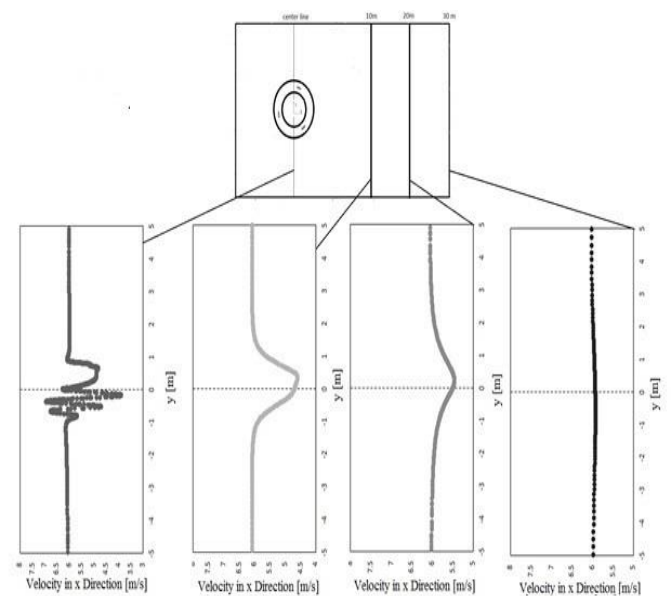

Fig. 10 Velocity magnitude at the centerline and 3 distances $(0$, $10,20 \mathrm{~m})$ at $\mathrm{TSR}=2$ and after 10 revolutions
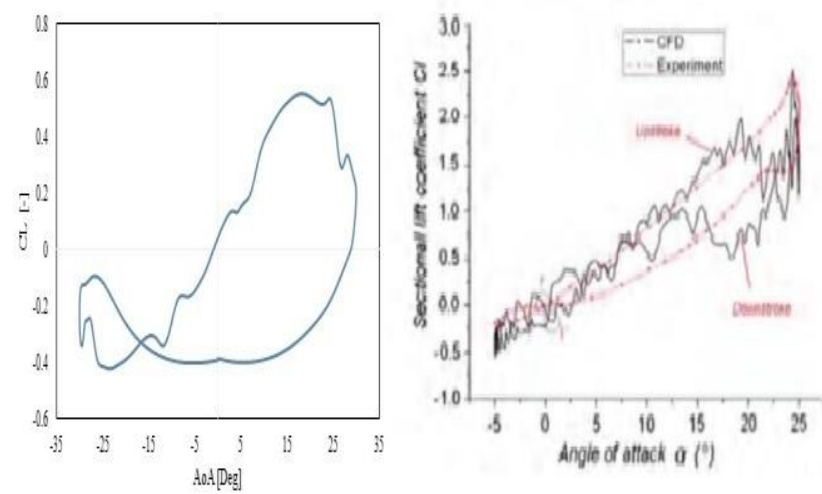

Fig. 11 Lift coefficient versus AoA at $\lambda=2$ a) SST $k-\omega$, and b) prior work (Wang et al. 2010, Lee and Gerontakos 2004 )

Four TSR values were used to extrapolate $\mathrm{Cp}$ versus $\lambda$ curves. Figure 12 compares the power coefficient obtained by this simulation with the experimental data from Lee and Gerontakos 2004. The $\mathrm{k}-\varepsilon$ model over predicts the power coefficient $(\mathrm{Cp})$ approximately $10.8 \%$; although, the SST $\mathrm{k}-\omega$ model presents a more accurate prediction, nearly $4.9 \%$, the SST k- $\omega$ results have a slight over prediction at the maximum point of power coefficient at TSR 2.6. This minor over estimation arises from 2D limitations and the existence dynamic stall (Biadgo et al. 2013). The maximum power coefficient is 0.34 , occurring when the TSR was equal to 2.6 .

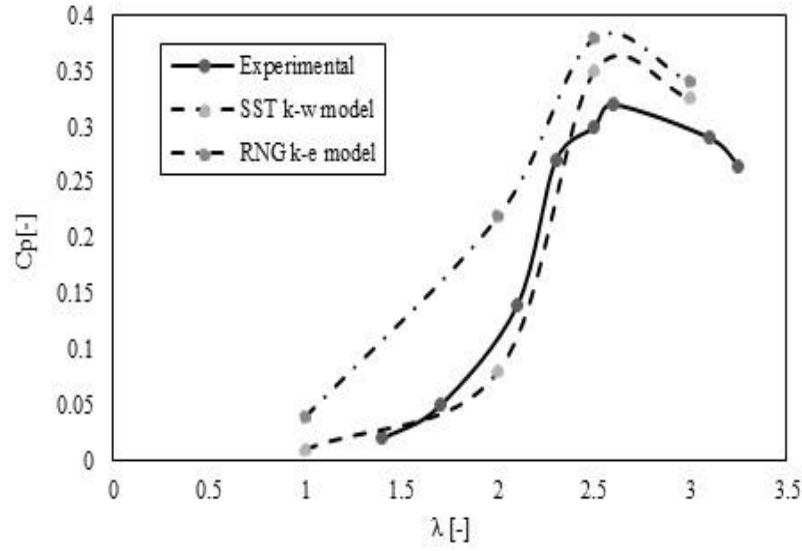

Fig. 12 Power coefficient $(\mathrm{Cp})$ versus TSR $(\lambda)$ compared with experimental data (RacitiCastelli, et al. 2011).

\section{Sensitivity Analysis}

\subsection{Effect of solidity factor}

According to previous studies in this field, solidity factor is the most effective parameter that effects on the flow behavior around Darius turbine. In this section, we consider three solidity factors in the range of low $(\sigma \leq 0.2)$, medium $(0.2<\sigma<0.4)$ and high $(\sigma>0.4)$. Effect of this parameter on Darius turbine performance will be discussed. Solidity factor is changed by changing the chord practical airfoil. Figure 13 and 14 present a comparison of CSFC and stream wise force coefficient (SWFC) for different solidity factor value. From figure 13 it can be seen that by increasing solidity factor from 0.12 to 0.25 , cross stream and stream wise forces would be double. Also by increasing it from 0.25 to 0.45 , cross stream force would be double and stream wise force would be about triple. Thrust and radial forces have a similar trend by increasing solidity factor (Figure 14).
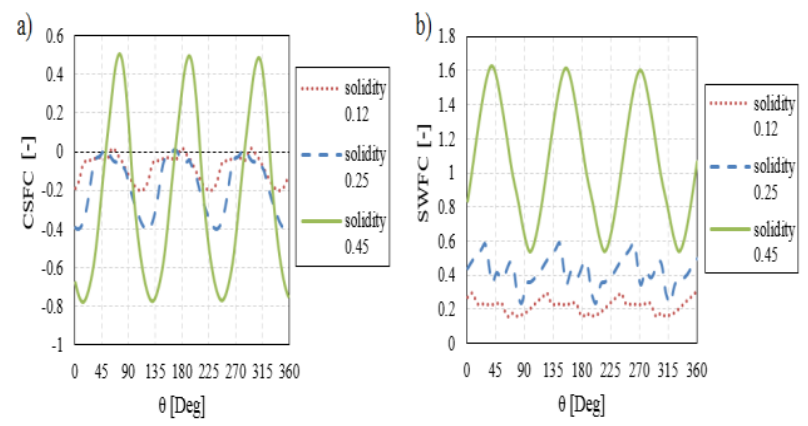

Fig. 13 Solidity factor effect on a) CSFC, and b) SWFC [ $\lambda=2$, NACA0021, $\left.V_{\infty}=6 \mathrm{~m} / \mathrm{s}\right]$

Figure 15 compares the power coefficient obtained in different solidity factor. Although at the tip speed ratio equal to 2 , aerodynamic forces of high solidity 
factor turbine are more than low solidity factor turbine (Figure $13 \& 14$ ). According to figure 15, power coefficient of low and medium solidity factor turbine is more than high solidity factor turbine. From this figure, it can be seen that the turbines with medium solidity factor show the best performance.
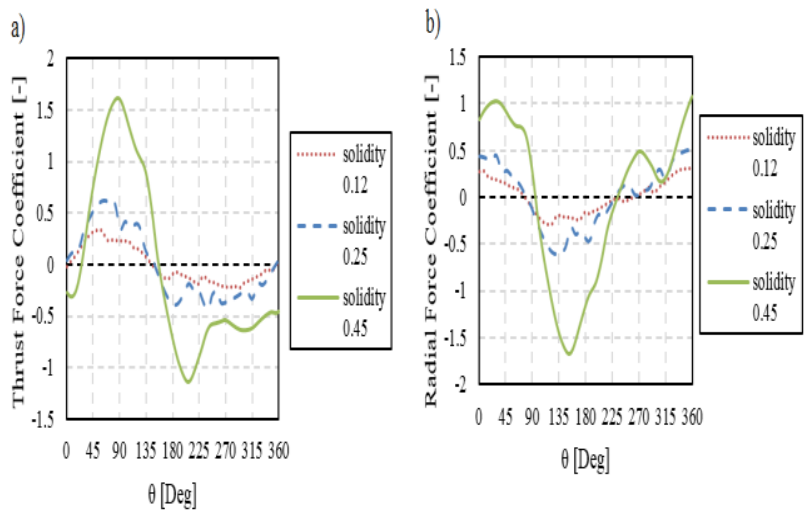

Fig. 14 Solidity factor effect on a) Thrust force coefficient, and b) Radial force coefficient [ $\lambda=2$, NACA0021, $V_{\infty}=6 \mathrm{~m} / \mathrm{s}$ ]

By increasing solidity factor, maximum power coefficient occurs in lower TSR and its value initially increases and then decreases. Maximum power coefficient of low solidity factor turbine is 0.33 and obtain at TSR equal to 3. Maximum power coefficient of medium solidity factor turbine, that is chosen as a base case study is 0.354 and occurs at TSR equal to 2.54. Finally, maximum power coefficient of high solidity factor Turbine is 0.31 and obtain at TSR equal to 1.5 .

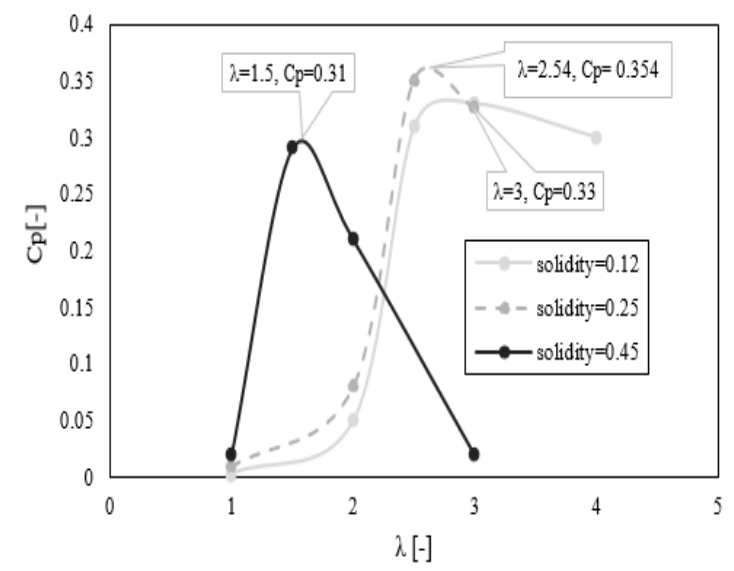

Fig. 15 Power coefficient $(\mathrm{Cp})$ versus TSR $(\lambda)$ at different solidity factor $\left[\lambda=2\right.$, NACA0021, $V_{\infty}=6 \mathrm{~m} / \mathrm{s}$ ]

\subsection{Effect of airfoil shape}

Airfoil shape, another effective parameter is used in this study. According to previous studies in this field, due to the anticipated maximum power factor and efficiency of NACA airfoil series have not much different with the advanced airfoil, and simple shape of them, often this airfoil type use in Darrieus turbine.
Figure 16 and 17 present a comparison of CSFC and SWFC for different airfoil shape. From these figures, it can be seen that NACA0012 thin airfoil behave differently than thick airfoil NACA0015 and NACA0021. Due to the stronger vortices would be seen by increasing the thickness of the airfoil at low TSR, in these TSRs force change domain would be increased. It is more tangible in the CSFC plot (Figure 16a). By analyzing these figures, fluctuations of turbine are detected that can play an important role in designing turbine and estimation of turbine useful life.
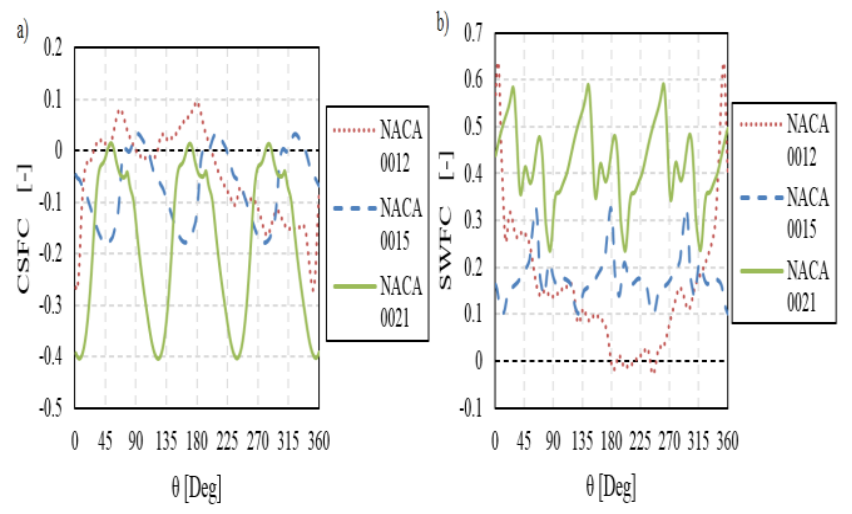

Fig. 16 airfoil shape effect on a) CSFC, and b)SWFC [ $\lambda=2$, $\left.V_{\infty}=6 \mathrm{~m} / \mathrm{s}\right]$
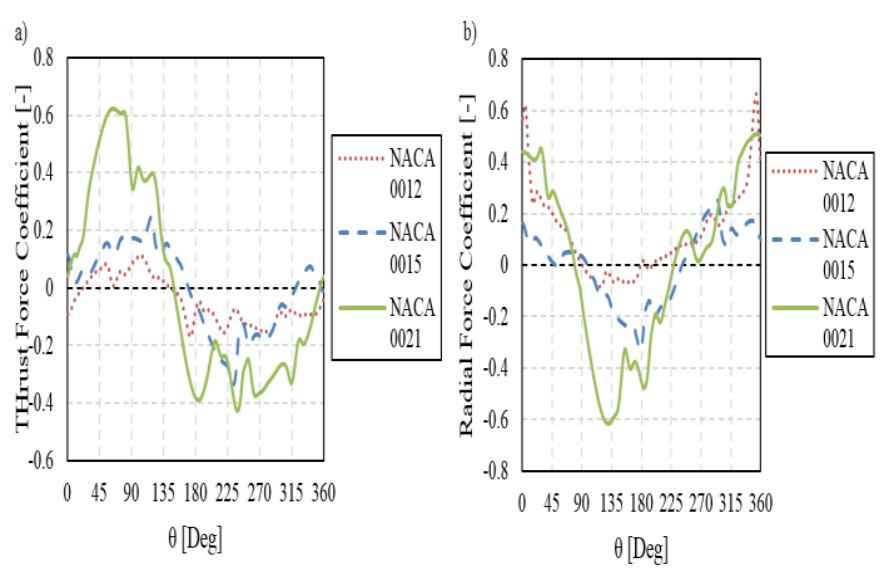

Fig. 17 airfoil shape effect on a) Thrust force coefficient, and b) Radial force coefficient $\left[\lambda=2, V_{\infty}=6 \mathrm{~m} / \mathrm{s}\right]$.

Figure18 compares the power coefficient obtained in different airfoil shape. Airfoil NACA0012, has less maximum power coefficient value $(\mathrm{Cp}=0.32)$ and this maximum value achieved at high TSR (TSR=3.3), while the maximum power coefficient value of NACA0015 and NACA0021 are not much difference and achieve at lower tip speed ratio. According to figure 18, NACA0015 obtain maximum power coefficient $(\mathrm{Cp}=0.349) \quad$ at TSR equal to 2.51 and NACA0021 obtain maximum power coefficient $(\mathrm{Cp}=$ 0.354 ) at TSR equal to 2.54 . 


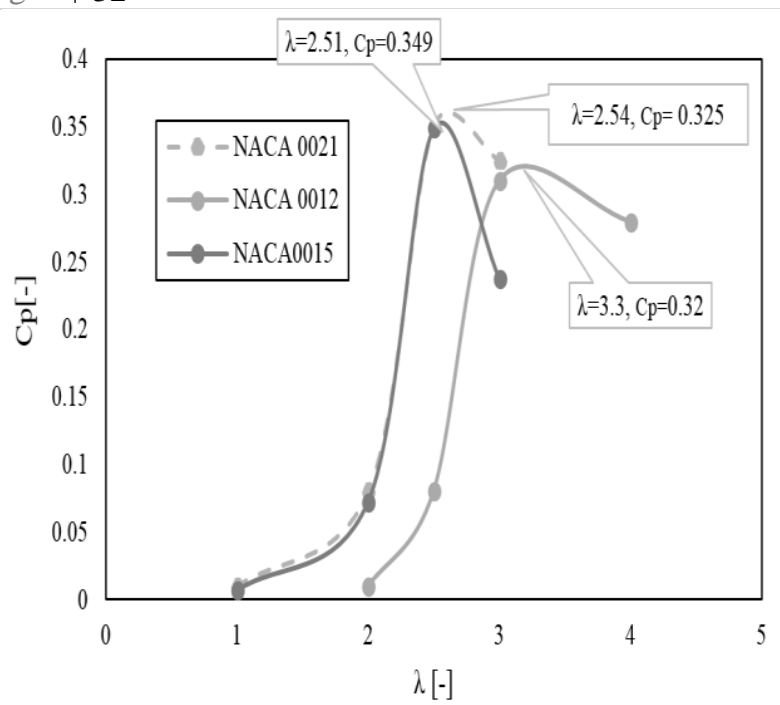

Fig. 18 Power coefficient $(\mathrm{Cp})$ versus TSR $(\lambda)$ at different airfoil shape $\left[\boldsymbol{\lambda}=\mathbf{2}, V_{\infty}=6 \mathrm{~m} / \mathrm{s}\right]$.

\subsection{Effect of wind speed}

Given that the aerodynamic forces are proportional to the square of wind speed and turbine power is proportional to the cubic of wind speed, this parameter has a significant role in turbine performance. Tehran's average annual wind speed is 3 $\mathrm{m} / \mathrm{s}$, and was chosen to evaluate the performance of the turbine. Figure 19 and 20 present a comparison of CSFC and SWFC for different wind speed. By changing the speed and increase from $3 \mathrm{~m} / \mathrm{s}$ to $6 \mathrm{~m} / \mathrm{s}$ don't see much change in the power, however, speed components of these speeds have $45^{\circ}$ phase differences that because of changing the behavior of turbines nearby flow and different angle vortex shedding arises. By changing wind speed from $6 \mathrm{~m} / \mathrm{s}$ to $9 \mathrm{~m} / \mathrm{s}$, the amount of force is almost 2.5 times.
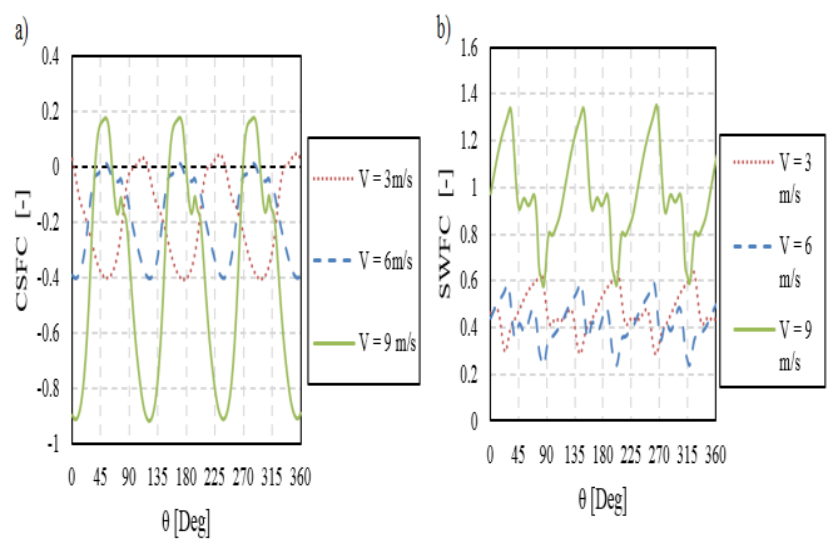

Fig. 19 Wind speed effect on a) CSFC, and b) SWFC [ $\lambda=2$, NACA0021]

Figure 21 compares the power coefficient obtained in different wind speed. According to figure 21, turbine at wind speed $3 \mathrm{~m} / \mathrm{s}$ obtain maximum power coefficient $(\mathrm{Cp}=0.22)$ at TSR equal to 3 . Whereas, at TSR equal to 1 , power coefficient at this wind speed is more than higher wind speed. Turbine at wind speed $6 \mathrm{~m} / \mathrm{s}$ and $9 \mathrm{~m} / \mathrm{s}$ respectively obtain maximum power coefficient $(\mathrm{Cp}=0.354$ and $\mathrm{Cp}=0.36)$ at TSR equal to 2.54 and 2.53. With increasing wind speed up to $6 \mathrm{~m} / \mathrm{s}$ power coefficient is a significant increase and then increase in this parameter is less evident. By reducing wind speed from $6 \mathrm{~m} / \mathrm{s}$ to $3 \mathrm{~m} / \mathrm{s}$, maximum power factor reduces by $38 \%$.

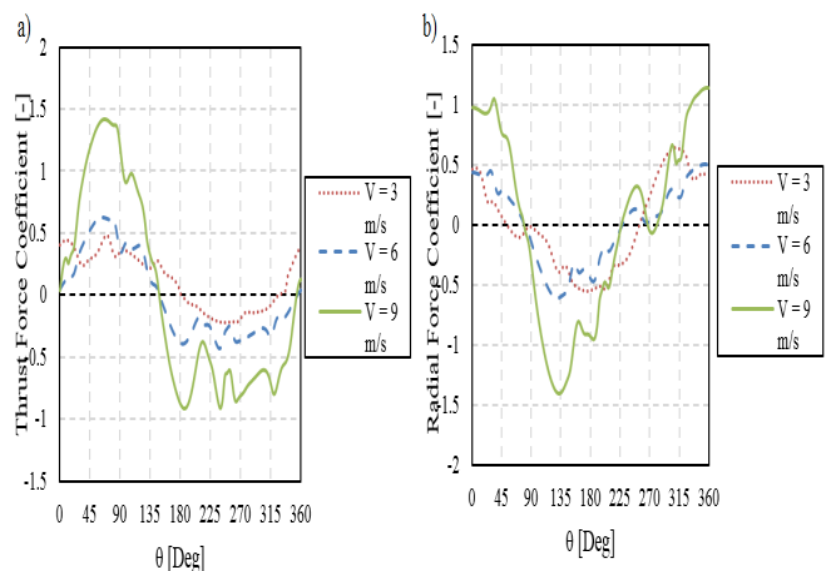

Fig. 20 Wind speed effect on a) Thrust force coefficient, and b) Radial force coefficient [ $\lambda=2$, NACA0021]

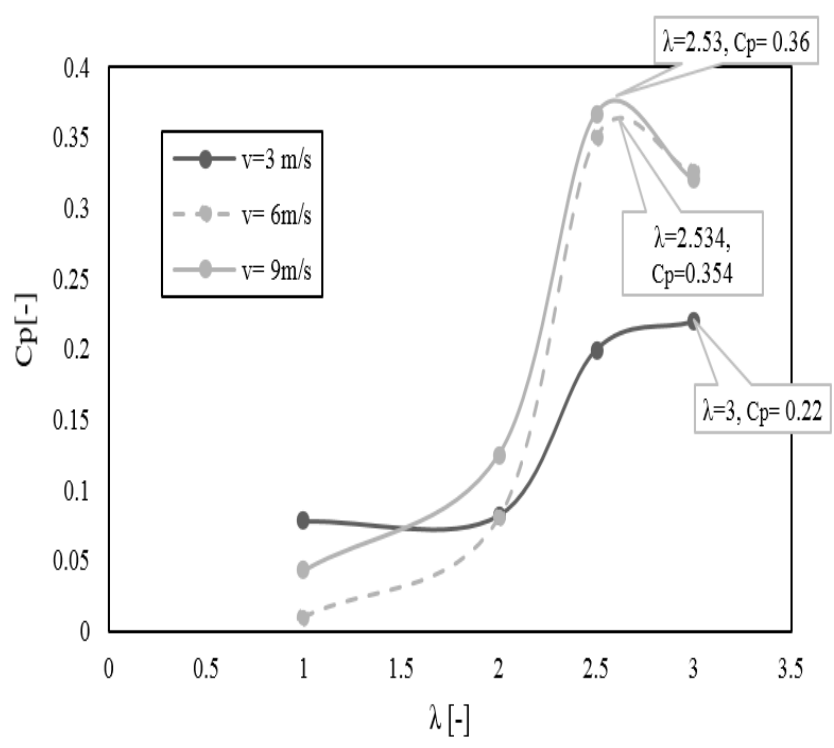

Fig. 21 Power coefficient $(\mathrm{Cp})$ versus TSR $(\lambda)$ at different wind speed $[\lambda=2$, NACA0021]

\subsection{Effect of strut}

To study the effect of strut on the performance of the turbine support structure, three modes without strut and a strut with a $3 \mathrm{~cm}$ diameter were considered. Figure 22 and 23 present a comparison of CSFC and stream wise force coefficient (SWFC) for different support structures. In figure 22 and 23, turbine aerodynamic forces values and their range could not be mapped. 

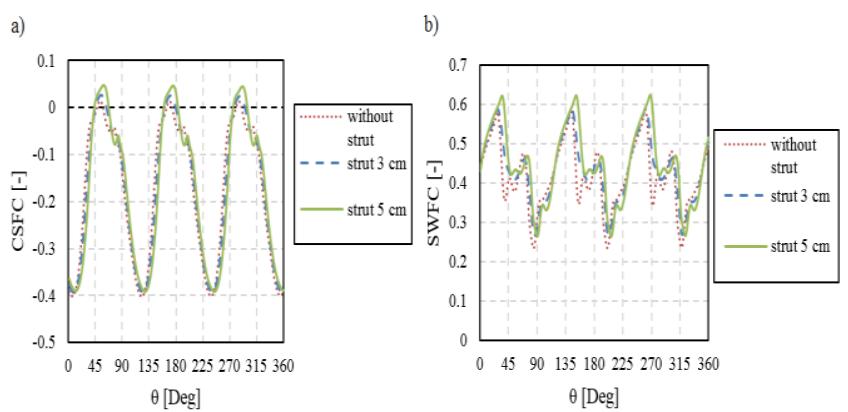

Fig. 22 Support structure effect on a) CSFC, and b) SWFC [ $\lambda=2$, NACA0021, $\left.V_{\infty}=6 \mathrm{~m} / \mathrm{s}\right]$

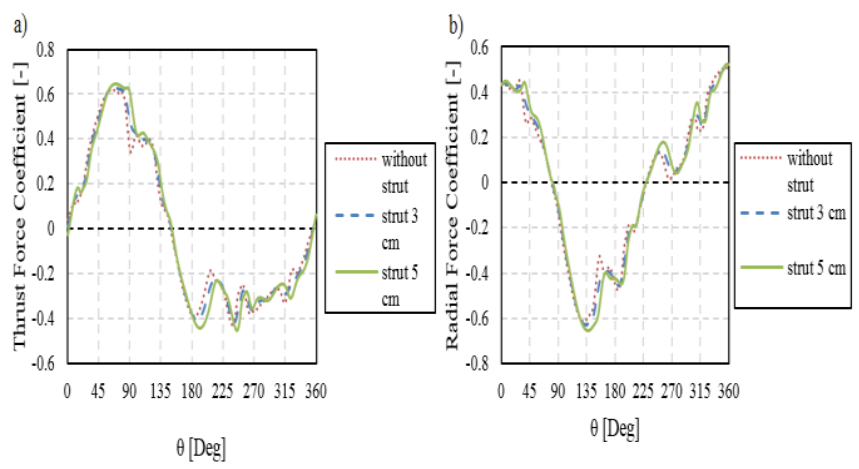

Fig. 23 Support structure effect on a) Thrust force coefficient, and b) Radial force coefficient [ $\lambda=2$, NACA0021, $V_{\infty}=6 \mathrm{~m} / \mathrm{s}$ ]

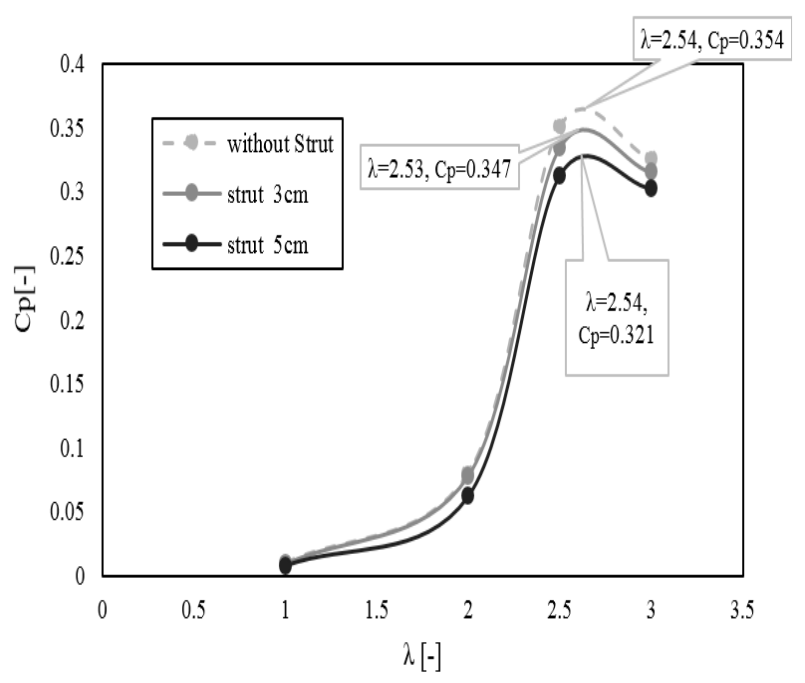

Fig. 24 Power coefficient $(\mathrm{Cp})$ versus TSR $(\lambda)$ with different support structure $\left[\lambda=2\right.$, NACA0021, $\left.V_{\infty}=6 \mathrm{~m} / \mathrm{s}\right]$.

Figure 24 compares the power coefficient obtained in different support structure conditions. According to this figure, it can be seen that by increasing the diameter of central shaft, power coefficient decreases and the maximum power coefficient occurs at the lower TSR. Maximum power coefficient of without strut condition is 0.354 while obtaining at TSR equal to 2.54. Maximum power coefficient of strut with 3 and $5 \mathrm{~cm}$ diameter conditions are 0.347 and 0.321 respectively. The maximum power coefficient loss by increasing the central shaft diameter from $3 \mathrm{~cm}$ to 5 $\mathrm{cm}$ is almost $8 \%$. The impact of these parameters compared to other parameters studied in the sensitivity analysis is not very noticeable.

\section{Conclusion}

Numerical simulations were carried out to estimate the aerodynamic performance of H-rotors Darrieus. In this simulation, flow behavior around the airfoil, such as the location of separation, dynamic stall angle, etc. were identified in detail, thus making this simulation useful for designing Darrieus turbines or analyzing vibration due to periodic loading. Simple 2D simulations were conducted by CFD code. The SST k$\omega$ model has been applied to simulate the flow conditions individually. The sliding mesh technique was adopted to handle the motion of the blades. The force coefficients obtained from the simulation showed a high level of agreement with the experimental data (RacitiCastelli, et al. 2011). The SST k- $\omega$ simulation at a TSR relative to the maximum power coefficient over predicted the power coefficient value by close to $4.9 \%$, because at this TSR value the dynamic stall has a considerable effect on aerodynamic loading. This study reveals that during a rotation, airfoil produced two vortexes consisting of a large leading edge vortex and small trailing edge vortex. The effect of the leading edge vortex can be seen by two peak points in the lift coefficient versus angle of attack curve. Three positive outcomes of this study consisted of measuring flow velocity in the wake, using SST k- $\omega$ turbulence model, and showing formation, shedding, and separation of the vortex in full rotation. Two limitations of this study consist of neglecting 3D effects and assuming a constant wind profile. Air velocities at different distances from the turbine were captured and the effect of the turbine in its wake as far as $30 \mathrm{~m}$ was observed. In the future, we plan to simulate the $3 \mathrm{D}$ flow around a Darrieus turbine and examine the 3D effects, like tip vortex, on the performance of the turbine.

Due to the results of solidity factor sensitivity analysis, by increasing solidity factor, maximum power coefficient occurs at lower TSRs. Medium solidity turbines have most maximum power coefficient than high and low solidity factor turbines. The results of the sensitivity analysis show that NACA0012 has a less maximum power coefficient than NACA0015 and NACA0021. This maximum power occurs at high TSRs. Maximum power coefficient of NACA0015 and NACA0021 does not show many differences; however, we can conclude that by increasing the airfoil thickness, power coefficient of turbines increases. Wind speed sensitivity analysis revealed by increasing wind speed, maximum power coefficient increases and occurs at lower TSR. This increase in changing speed from $6 \mathrm{~m} / \mathrm{s}$ to $9 \mathrm{~m} / \mathrm{s}$ is significant. Finally, the effect of the central shaft diameter in order to evaluate support structure effects 

Renewable Energy Development, 7(1),23-34, doi.org/10.14710/ijred.7.1.23-34

$\mathrm{P}$ a g e $\mid 34$

reveal this parameter has no impact on turbine power coefficient.

\section{Nomenclature}

A

$\mathrm{C}_{\mathrm{d}}$

$\mathrm{C}_{1}$

$\mathrm{C}_{\mathrm{n}}$

$\mathrm{Cp}$

CSFC

C

DES

DMSM

$\mathrm{F}_{\mathrm{n}}$

$\mathrm{F}_{\mathrm{t}}$

$\mathrm{F}_{\mathrm{X}}$

$\mathrm{F}_{\mathrm{y}}$

$G_{b}$

$G_{k}$

$\tilde{G}_{k}$

$G_{\omega}$

$\mathrm{L}$

LES

$\mathrm{K}$

$\mathrm{N}$

$\mathrm{N}$

PISO

$\mathrm{R}$

Re

RNG

SWFC

SST

$S_{k}$

S.

$\mathrm{T}$

$\overline{\mathrm{u}_{2}}$

ú,

$u_{E f f}$

URANS

V.

$\mathrm{Y}$

$\mathrm{Y}+$

$Y_{k}$

$\mathrm{Y}_{\mathrm{w}}$

$\alpha(\mathrm{AoA})$

$\alpha_{x}$

$\alpha_{\mathrm{k}}$

$\theta$

2

$\mu$

pứ:í,

$\sigma$

Cross-sectional area of the wind turbine

Drag coefficient [-]

Lift coefficient [-]

Normal force coefficient[-]

Mean power coefficient [-]

Thrust force coefficient [-]

Cross Stream Force Coefficient

Chord [m]

Drag force

Detached Eddy Simulation

Double Multiple Streamtube Model

Normal force

Tangential force

$\mathrm{x}$ direction force

$\mathrm{y}$ direction force

The generation of turbulence kinetic energy

The generation of turbulent kinetic energy

The generation of turbulent kinetic energy

The generation of $\omega$

Lift force

Large Eddy Simulation

Turbulence kinetic energy [-]

Number of blades [-]

Rotational speed [r/min]

the Pressure-Implicit with Splitting of Operators

Rotor radius [m]

Reynolds Number [-]

Reynolds-Normalization Group

Stream wise force coefficient [-]

Shear Stress Transport

User define source terms

User define source terms

Time [s]

Mean velocity component

The fluctuating velocity components

Eddy viscosity

Unsteady Reynolds-averaged Navier-Stokes

Wind speed $[\mathrm{m} / \mathrm{s}]$

Distance from surface [m]

Dimensionless wall distance [-]

The dissipation of $\mathrm{k}$

The dissipation of $\omega$

Angle of Attack [deg]

Inverse effective Prandtl numbers for $\varepsilon$

Inverse effective Prandtl numbers for $\mathrm{k}$

Dissipation [-]

Azimuth angle

Tip speed ratio [-]

Viscosity

Density

The Reynolds stresses

Solidity [-]

$\begin{array}{ll}\text { Tw } & \text { The Wall Shear Stress } \\ \phi & \text { Scalar } \\ \varpi & \text { Specific dissipation rate [-] } \\ \Gamma_{k} & \text { The effective diffusivity of k } \\ \Gamma_{\omega} & \text { The effective diffusivity of } \omega\end{array}$

\section{References}

Allet, A., Paraschivoiu, L (1995) Viscous Flow and Dynamic Stall Effects on Vertical-Axis Wind Turbines. International Journal of Rotating Machinery, 2(1), 1-14.

Betz, A. (1920) Das Maximum der theoretisch möglichen Ausnützung des Windes durch Windmotoren. Zeitschriftfür das gesamte Turbinenwesen. 26, 307-309.

Biadgo A. M., Simonovic, A., Komarov, D., \& Stupar, S. (2013) Numerical and Analytical Investigation of Vertical Axis Wind Turbine. FME Transactions, 41, 49-58.

El-Samanoudy, M., Ghorab, A. A. E., \& Youssef, S. Z. (2010) Effect of some design parameters on the performance of a Giromill vertical axis wind turbine. AinShams Engineering Journal, 1(1), 85-95.

Hamada, K. (2008) Investigation of scale economies for African biogas field study. Solar Energy, 82(4), 911-917.

Hwang, L. S., Lee, Y. H., \& Kim, S. J. (2009) Optimization of cycloidal water turbine and the performance improvement by individual blade control. Applied Energy, 86(9), 15321540 .

Horiuchi, K., Ushiyama, I., \& Seki, K. (2005) Straight wing vertical axis wind turbines: A flow analysis. Wind Engineering, 29(3), 243-252.

Howell, R. (2010) Wind tunnel and numerical study of a small vertical axis wind turbine. Renewable Energy, 35(2), 412422.

Guerri, O., Sakout, A., \& Bouhadef, K. (2007) Unsteady flow simulation and dynamic stall around vertical axis wind turbine blades. AIAA aerodynamics conference Reno.

Launder, B. E., \& Spalding, D. B. (1972) Lectures in Mathematical Models of Turbulence. Academic Press, London, England.

Lee, T., \& Gerontakos, P. (2004) Investigation of flow over an oscillating airfoil. Journal of Fluid Mechanics, 512, 313-341.

Menter, F. R., Kuntz, M., \& Langtry, R. (1990) Ten years of industrial experience with the SST turbulence model. Flow Turbulence and Combustion, 77, 277-303.

RacitiCastelli, M., Englaro, A., \& Benini, E., (2011) Darrieus wind turbine: proposal for a newperformance prediction model based on CFD. Energy, 36(8), 4919-4934.

Spera, D. (2009) Wind Turbine Technology, fundamental concepts of wind turbine engineering. ASME Press.

Wang, S., Ingham, D. B., Ma, L., Pourkashanian, M., Tao, Z. (2010) Numerical investigations on dynamic stall of low Reynolds number flow around oscillating airfoils Computers \& Fluids, 39(9), 1529-1541.

White, M. F. Fluid mechanics. Ed. McGraw-hill. 